\title{
A Case of Application of Data Transforms
}

\author{
SEAD SPUZIC \\ STEM \\ The University of South Australia \\ Level 1, 101 Currie Street, Adelaide SA 5000 \\ AUSTRALIA
}

\begin{abstract}
The recent implementations of Industry 4.0 and allied mathematical applications such as machine learning and big data analytics are conditioned by mathematizing the basic features of the observed system. For example, the key phenomena in a number of man-made processes are controlled by an orifice, an opening through which is passing a medium of interest. When the observed process is recursive, the related records indicate the possibility of extracting from the accumulating observations knowledge useful for the system optimisation. Many of the process variables such as chemical composition, velocities, temperatures, and forces, are recorded in a convenient digital format. This, however, is not always the case with the orifice geometry. Mathematical transforms presented hereby demonstrate how a broad variety of the orifice geometries can be defined in a generic mathematical format that allows for analysing them within the same observation space.
\end{abstract}

Keywords: - Chebyshev Transforms, Orifice, 2D Geometry, Vectorization, Digitizing, Big Data Analytics

Received: January 3, 2021. Revised: July 4, 2021. Accepted: July 20, 2021. Published: July 27, 2021.

\section{Introduction}

The efforts to optimize the man-made systems, especially the recursive industrial processes such as agricultural, transportation and metalworking operations, contribute significantly to advancements in information sciences. A typical example is Big Data Analytics that is widely recognized as a paradigm useful for further developments in information theory. $[1,2]$

While the volumes of records apparently present no issues, when it comes to knowledge extraction, the format of records can significantly limit the range of the observation (sample) space. Digitized observations, the most convenient sets for statistical analyses, can fall within incompatible groups despite the need for analyzing them within the same observation space. There is the risk of wasting all the value and wealth of information contained on these databases unless there are used the adequate techniques to ensure data completeness. [3-5]

For example, the effect of orifice geometry is of much interest in numerous industrial systems. However, the presently published analyses are limited to examining the cases of simple geometries such as square, rectangular, round, and oval. The present metrics makes it impossible to take into account the broader diversity of orifice geometries.

The studies including such simple geometries are carried out to analyse the effect of orifice geometry on the pressure profile of a passing fluid [6,7]. The published findings fell short of addressing the possible effects of the refined modifications in the contour elements indicated in Fig. 1.

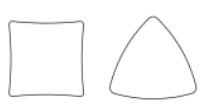

Fig. 1: Examples of possible modifications of the orifice geometry, not explored in $[6,7]$

The authors in [8] explored the effect of the orifice geometry on the acoustic impedance. Exploring the influence of each of the different geometries presented in Fig. 2 is affected by the format of how these geometries were defined.

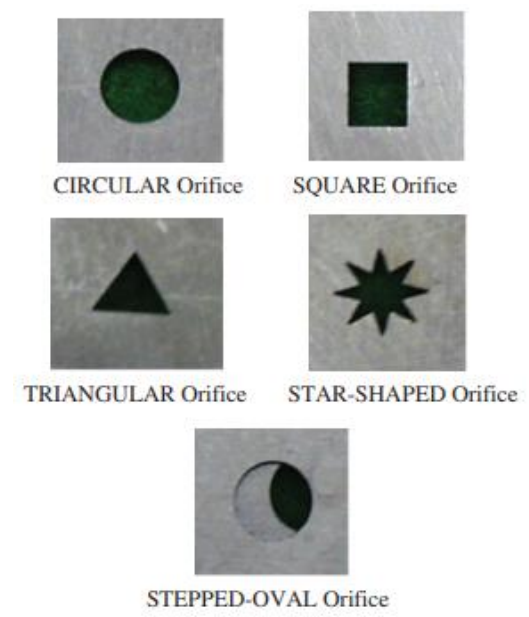

Fig. 2: The variety of orifices used to examine the effect of geometry on the acoustic impedance [8] 
However, the authors in [8] could not correlate the investigated effect to the geometry other than to point which one out of 5 options is most or least influential. If the input data were prepared by introducing a common generic vector, the gradual modifications between these geometries could be expressed by means of changing the values of such generic vectors. The authors do not know whether the optimum impedance is in fact some halfway between the two or three investigated options.

The analogous drawback is observed in the propulsion system analyses carried out to investigate the effect of the plainest nozzle geometries (circular, elliptical, square, and triangular) on the patterns of the standing waves. [9]

The examples in Figures 3 to 6 illustrate even more diverse geometries that can be found in the tool assemblies used to manufacture long solids of a wide range of cross-sectional shapes by applying plastic deformation by means of drawing and extrusion $[10,11,12]$. The products shown in Fig. 3 are manufactured by squeezing a sold material through an orifice thus forcing the plastic deformation and changing the initial cross-section (usually rectangular or round) into the newly formed cross-section of the product. The orifice contour is identical to the newly produced cross-section (except for a minor effect of the elastic recovery).

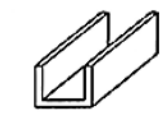

Channels

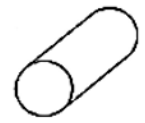

Round Bars

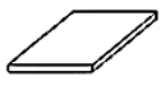

Bars

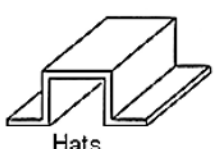

Hats

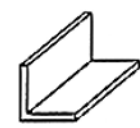

Angles

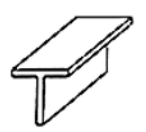

Tee Bars

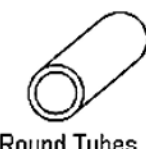

Round Tubes

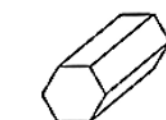

Hexagon

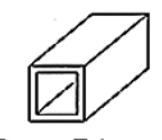

Square Tubes

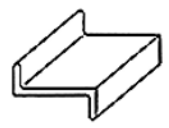

Zee Bars

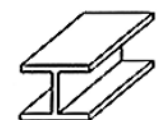

I-Beams

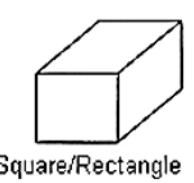

Fig. 3: Selected examples of cross-sections manufactured by extrusion or drawing
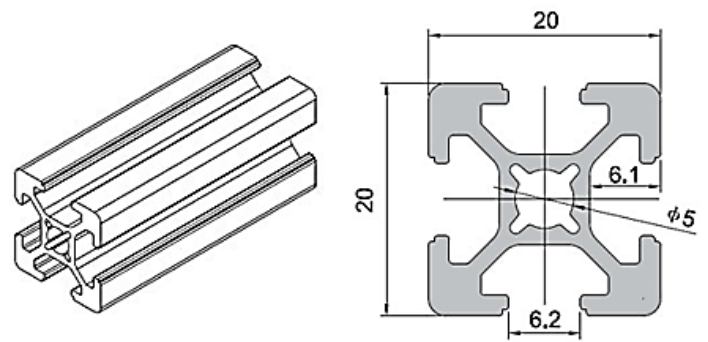

Fig. 4: More complex extrusion geometries [13]
The variety of geometries that are used in rolling technology is even wider due to the nature of the forming process: the final product is obtained after shaping the product cross-section in a number of successive passes, Figures 5 to 8 .

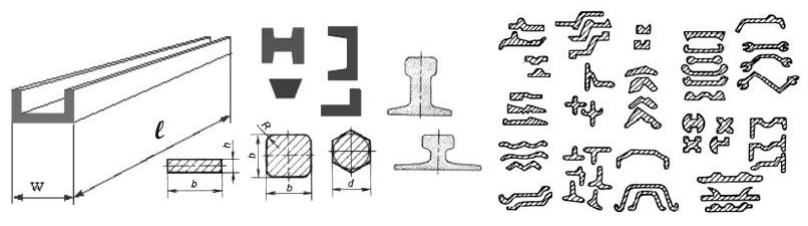

Fig. 5: Variety of geometries in rolling technology

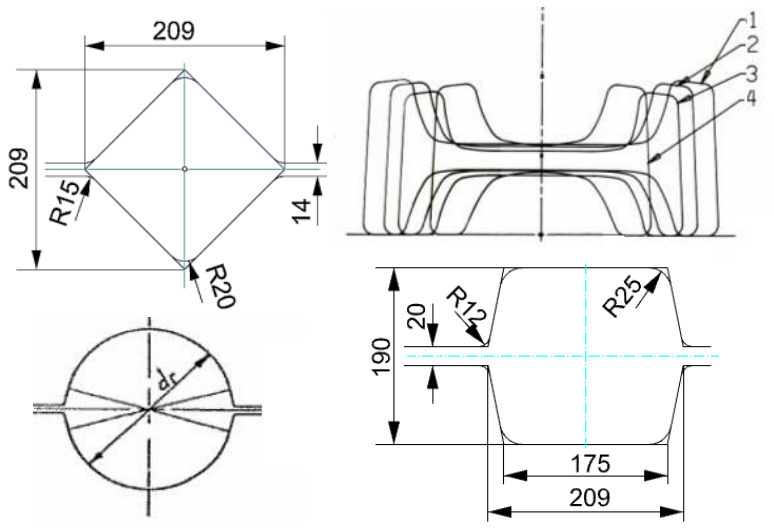

Fig. 6: Variety of the orifices in rolling technology

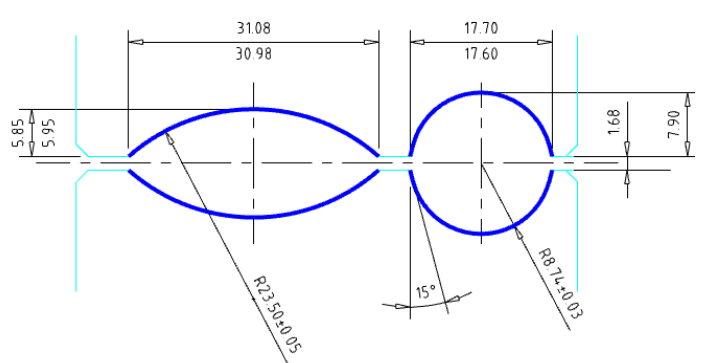

Fig. 7: Examples of double symmetrical orifices used in rolling passes.

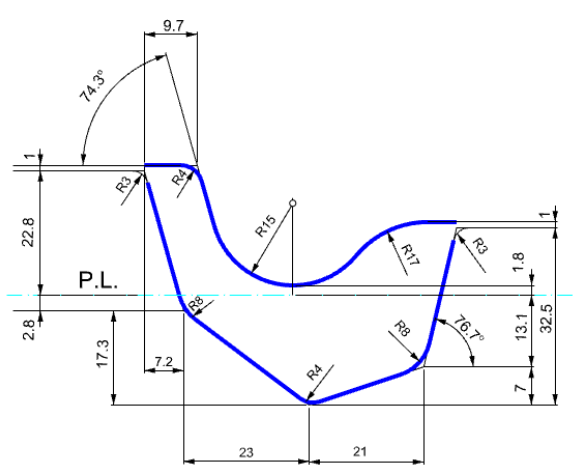

Fig. 8: Example of asymmetrical orifice used in rolling process 
In the case of rolling the orifice is not embedded in a static tool; the orifice contour is delimited by the surfaces of two, three, or four solids of revolution - the rollers (in rolling practice the term 'rolls' is in frequent use). The grooves embedded in the roll surface create the orifice contour. The rolls are rotating around axes that are kept in a specific position thus forming the required orifice. [14]

In this manuscript, an algorithm for transforming the otherwise 'incompatible' 2D contours, into generic mathematical form is described. The proposed mathematical approach is applicable for data structuring and preparation for each of the different orifice applications. The resulting generic vectors allow for applications of advanced methods such as machine learning and data mining within each of the separated technological domains.

Developing this transform is not motivated by searching for correlations between the orifices used in plastic forming and those used in the fluid dispersion. The motive is to present a practical mathematical transform useful for discovering new knowledge that is specific within the boundaries of each of these different technologies.

\section{Problem Formulation}

From the technological point of view, the problem is how to create more comprehensive knowledge about the effect of the orifice (tool) geometry on the key performance indicators, as well as the knowledge about the interactions between the geometry and other technological data (such as temperature, velocity, chemical composition, etc.). These orifices enable the ultimate function of the surrounding complex system. Designers can increase the complexity and costs of the jet engine propulsion system controls and frames, nevertheless, it is the reliability of the exhaust nozzle that warrants the aircraft trust and the control valve function. Technologists in a hot rolling mill can introduce the most expensive high-temperatureresistant high-speed steels as the forming tools, yet, if the grooves that are exposed to the rolling-sliding contact with the processed materials wear too fast due to poor design of the orifice geometry, the product will fall out of the tolerance limits

From the Data Science points of view, the existing metrics used to define the orifice geometry are not well adjusted for analyses and optimisation of the interactions with other variables of processes utilising orifices. In particular, the precise data about the geometric intricacies are not prepared, structured, and digitized in a format that embraces sufficiently broad variety. Therefore, the advanced mathematical methods cannot be applied to discover the important patterns and correlations and cannot use that knowledge to further optimise the orifice geometry.

From the mathematical point of view, the problem can be presented as follows: there is a need to transform the curve geometry so it can be defined as a generic vector. The values of the vector components must be real numbers. The range and the order of the magnitude of these numbers must allow for numerical and statistical analyses of the sufficiently broad variety of curves of the practical interest. This variety presents a set of closed curves defined in 2D space and satisfying the following limitations:

- the curve can be broken into a sub-set of osculating arcs,

- the curve does not compromise knots,

- the curve length is a real number.

Also, the procedure for a reverse one-to-one translation from the orifice-contour-vector to the initial curve must be defined.

Depending on the practical application, the vector dimension is to be minimized while embracing the broadest possible variety of geometries of significance. Nonetheless, this final limitation is released dramatically by the contemporary development in the Big Data acquisition and processing systems that enable analysing within real-time extremely large data sets.

\section{Solution}

\subsection{Prelude}

The solution requires the positioning of each curve in the same coordinate system. The positioning of the double symmetrical closed curves has a straightforward solution as long as one-to-one mapping of the $x$ and $y$ coordinates can be realized for each point. For the double symmetrical cases shown in Figures 1, 2 (except for the star-shaped orifice), 3 (except for the $\mathrm{Z}$ and $\mathrm{T}$ bars), 6 , and 7, the contour centre of the gravity coincides with the coordinate origin $(0 ; 0)$ and hence, it is sufficient to define the contour segment falling in the first quadrant only.

The star-shaped orifice shown in Fig. 2 and the orifice shown in Fig. 4 do not satisfy the condition of one-to-one mapping in the $y-x$ coordinate system. The contour centre coincides with the coordinate origin $(0 ; 0)$, nonetheless, the curve belonging to the first quadrant needs to be modified before finally transforming it into the generic vector $\mathbf{A}$. 
And, finally, in the case of the orifices with a single axis of symmetry, and for the asymmetrical orifices, a standardized method needs to be defined for positioning such curves in the coordinate system. The $\mathrm{T}$ and $\mathrm{Z}$ geometries shown in Fig. 3, and the shape in Fig. 8 belong to this category, along with a practically infinite variety of additional geometries.

While the orifice cross-sections used in the processes of drawing and the extrusion are the closed curves, the orifices in rolling (so-called calibres) are formed with two, three or four separate curves. For the sake of simplicity, in this manuscript, only the case of two curves will be discussed. Examples of such orifices are illustrated in Figures 6, 7 and 8. It is apparent that the orifice curves are brought into a specific position and are separated by a certain narrow gap. The nature of the rolling pass is such that the major portion of the processed material cross-section will assume the shape following the 'top' and the 'bottom' curve. At the same time, the contour fragment of the small segment that fills in the gap portion is assuming the form governed by the plastic flow of the processed solid. With having this in mind, the imaginary closed orifice contour can be defined by the crosssection contour of the rolled material.

\subsection{Double symmetrical case - simple shape} This group of orifices includes the geometries that can be represented in the first quadrant by means of an analytic function that satisfies the condition of the one-to-one $y-x$ mapping.

It is required that varying the coefficients of the function $y(x)$ results in a spectrum of curves of interest. The ordered tuples of the $y(x)$ coefficients are the components of vector $\mathbf{A}$ thus representing the digitized format for the observed orifice contour.

The curve fitting based on Chebyshev polynomials $[15,16]$ provides a satisfactory method for digitizing such geometries, Eqn. (1) and (2):

$$
\begin{gathered}
T_{n}(\xi)=\cos (n \cdot \operatorname{acos}(\xi)) \\
0 \leq \xi \leq 1
\end{gathered}
$$

The vector $\mathbf{A}$ is defined by the components $a_{n}$ mapped from Eqn. (3):

$$
\sum_{n=0}^{N} a_{n} T_{n}(\xi)
$$

where:

$n=$ positive integer

$\xi=$ real number

$N=$ positive integer (the vector dimension).

Additional transformation allows for further normalisation of the contour database. As long as the one-to-one mapping is preserved, the simple geometries can be translated in the $y-\alpha$ coordinate system, as defined by Equation (4) and Figure 9.
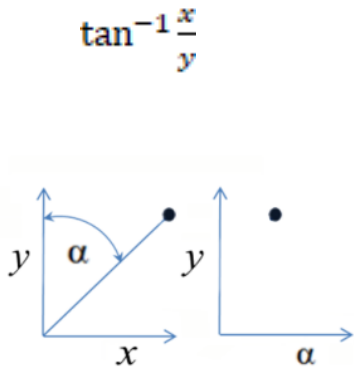

Fig. 9: Example of the $y-x$ to $y-\alpha$ transform

Some examples of the orifices suitable for the conversions using Eqn. (4) include rectangular, round, oval, the cases shown in Fig. 1, etc.

The order of the Chebyshev polynomials should be selected to secure the sufficient precision of the contour derived backward from vector A. For example, the orifices presented in Fig. 7 were successfully analyzed by means of 12-dimensional vectors derived from the Chebyshev polynomials defined in Equations (3) and (4) with $N=11$. [16]

\subsection{Double symmetrical complex shape}

This group consists of double-symmetric geometries (such as shown in Fig. 10) that cannot be simplified using Eqn. (4) and extends to the further complex double-symmetric shapes which cannot be represented in the first quadrant by means of analytic functions that satisfy the condition of the one-to-one $\mathrm{y}-\mathrm{x}$ mapping (see Figures 4 and 11).

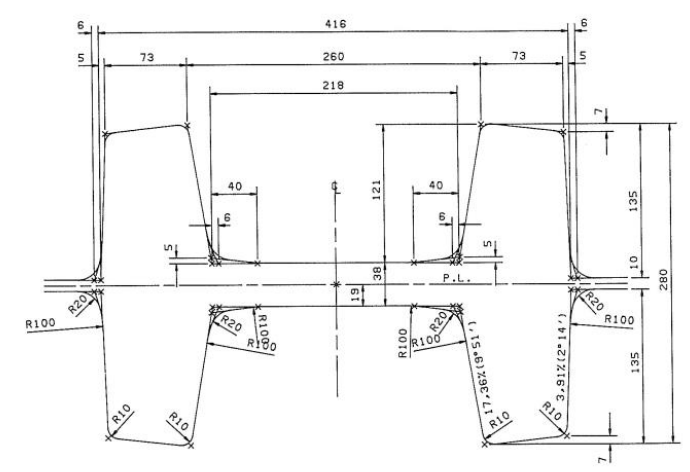

Fig. 10: An example of the tool contour used in the intermediate passes for manufacturing structural sections by rolling 


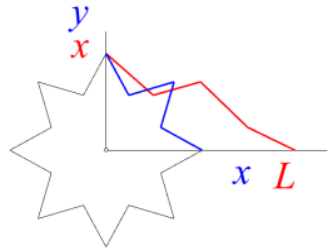

Fig. 11: Conversion of a double-symmetrical shape from $y-x$ to $x-L$ coordinates

Figure 10 shows an orifice created by the grooves cut in two solids of revolution (so-called 'rollers', or 'rolls' [14]) each. The rolls are positioned one above the other and their axes are mutually parallel. This configuration is defined by the cross-sections of the groove contours with the plane passing through both axes, as shown in Fig. 10. It is obvious that a vector departing from the origin to point at the top-right corner (the fillet of a radius R10) would generate three points following the Eqn. (4). This violates the one-to-one mapping constraint, i.e. the orifice cannot be included in the group of interest.

The positioning of the orifice contour in the coordinate system is resolved automatically at the intercept of the axes of symmetry. However, there is a need to introduce an additional translation step: conversion from the $y-x$ coordinates into $x-L$ coordinates, as demonstrated in Fig. 11.

The contour length $L$ values are measured backward, starting from the intercept of the contour with the $x$-axis (in the first quadrant of the $y-x$ system).

For the classes of the orifices where the reverse transform from $x-L$ into $y-x$ coordinates results in multiple solutions, such as shown in Fig. 12, additional components need to be introduced: the surface area of the orifice within the $y-x$ coordinate system.

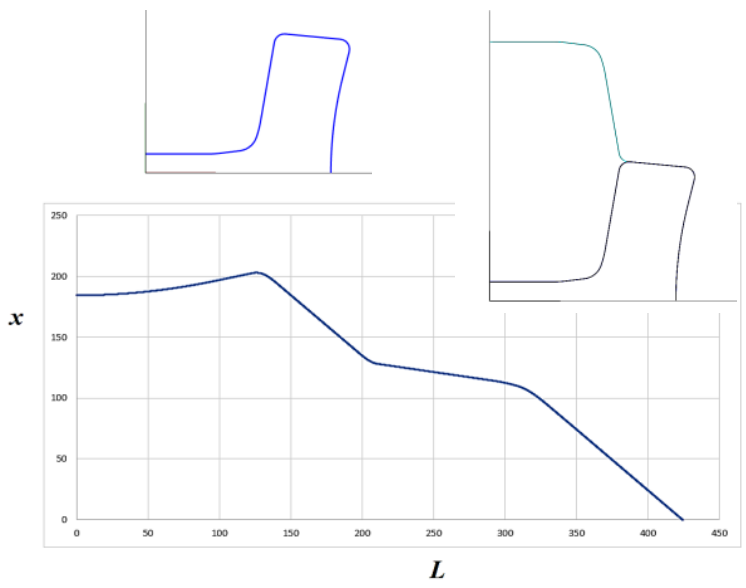

Fig. 12: The stages of two-way translating the doble-symmetric contour between the $y-x$ and $x-L$ coordinate system
The top-left graph in Fig 12 shows the contour of the double-symmetric orifice in the first quadrant of the $y-x$ system. At the bottom is shown the same contour in the $x-L$ coordinate system. The result of the reverse translation into the $y-x$ coordinates is shown on the top right portion of Fig. 12 (the lightblue curve is rejected since it failed the area test).

The information about the orifice area allows for programming the criteria for eliminating incorrect options. Basically, the computer program calculates all possible contour paths and then selects the one that matches the correct area.

The Chebyshev transforms are chosen because they provide the optimum solution for polynomial interpolations. The roots of $T_{n}(x)$ (also called Chebyshev nodes) are used as matching points for generating the two-dimensional NURBS splines. NURBS (Non-Uniform Rational B-Splines), are mathematical representations of geometry that can accurately describe any shape from a simple 2D line, circle, arc, or other osculating curves. The Chebyshev polynomial-based method provides more appropriate curve fitting than the other curve fitting techniques such as the least squares method since it prevents extensive oscillations of the fitting function [15]. Moreover, the method provides the rule for selecting the sampling points - the Chebyshev nodes, as demonstrated in detail in [16].

The problem of capturing the curve regions with the delicate geometry is resolved by superimposing two, or even more polynomials on the same contour. The connect-the-dots process corresponds to the task of reconstructing the connectivity of a planar curve from a set of structured points sampled on that curve.

A sparser sampling is beneficial for this leads to constructing the generic vectors with fewer components. Nonetheless, when is desirable to employ a method that covers a larger variety of curves, it is inevitable that some fragmenting of the contour becomes involved.

Figure 13 illustrates a case when there is a need for capturing a local segment defined by the arc of radius $R_{3}$.

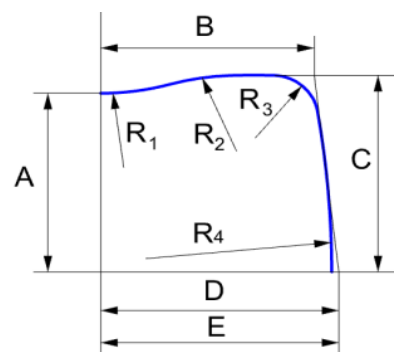

Fig. 13: The $1^{\text {st }}$ quadrant presentation of the doublesymmetrical contour with intricate curvature 
This class of geometries can be captured by defining the Chebyshev nodes in two stages, each employing the Chebyshev polynomials of the order 20, as shown in Figures 14 and 15.

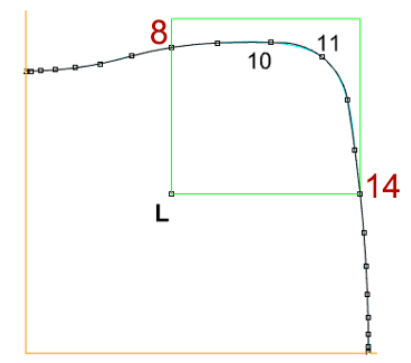

Fig. 14: The first phase of curve fitting

The first phase of the Chebyshev transform (polynomial order 20) that is shown in Fig. 14 produces a sufficiently precise fit (the error is less than $0.03 \mathrm{~mm}$ ) only beyond the green square. As discussed above, the robust procedure for defining the Chebyshev nodes provides a generic rule applicable for selecting the set of reference points on an arbitrary curve. The reverse calculations of these points using Eqn. (3) result in precisely reproducing the original points. However, the reverse reproduced curve, oscillates between the nodes thus resulting in some deviation from the original contour. The density of the Chebyshev nodes is decreasing with the distance from either of the contour ends (intercepts with the coordinates in the first quadrant). For a type of contour shown in Fig. 13, the scarce distribution of the nodes allocated to the segment at the radius $\mathrm{R}_{3}$ allows for increased oscillations between the nodes that are far apart. Consequently, the first phase is not sufficient, since the deviation between the reproduced and original curve is too large within the green frame in Fig. 14.

Therefore, for capturing the curve segment within the green square, the $2^{\text {nd }}$ stage is performed using again the Chebyshev polynomial of the order 20, as shown in Figure 15. The coordinate origin is shifted to the point $\mathrm{L}$ which is, in this example, defined by the Chebyshev nodes 8 and 14 .

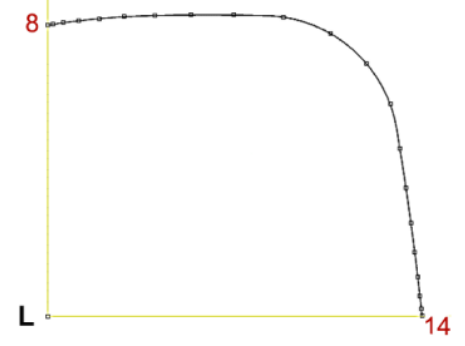

Fig. 15: The second phase of the curve fitting
During the reverse translation, the segments of the curves obtained in Figures 14 and 15 are merged thus producing a fit with satisfactory precision. The corresponding vector has 42 components.

The cutting points in Figure 14 that are used to produce contour in Figure 15 are nodes No. 8 and No. 14. The intercept of the line passing through point 8 and is parallel to the ordinate and the line passing through point 14 and is parallel to the abscissa defines the new coordinate origin $(0,0)$.

In the case that there is a need to cover even more complicated cases the order of the Chebyshev polynomials or the number of the superimposed segments needs to be increased and the cutting points re-defined.

\subsection{Asymmetric cases}

The solution requires the positioning of each curve in the same coordinate system by applying an appropriate standardized procedure. In other words, a uniform procedure is required for deciding where exactly is the position of the orifice contour in the coordinate system, including the orientation (angle) relative to the ordinates.

In an overwhelming majority of the extrusion and drawing processes, the starting cross-section is double-symmetric, i.e. a rectangle or a circle. When the starting geometry is rectangular, the resulting asymmetric contour will position itself (translate and rotate) following the lowest resistance to the solid flow. The location in the coordinate system of such translated and rotated orifice contour is found by superimposing it over the axes of the entering rectangular section. When the entry contour is a circle, the rotation is omitted - the exiting contour is kept at the technologically defined position.

The rolling process is conducted through a series of subsequent passes, each being realized in a different orifice. The correlation between the orifices in the sequence of passes is of much interest in rolling technology.

The contour in Fig. 8 shows a technical drawing of an asymmetric orifice used in the rolling process. It is apparent that all dimensions are related to the so-called pitch line (P.L.), which is the line in the middle of and parallel to the roll axes.

Although positioning the pitch line on the horizontal coordinate seems a straightforward solution, more information will be captured if the 'imaginary orifice' from the antecedent pass is used as the frame for orienting the observed contour.

By going backward along with the series of passes, a double-symmetrical contour is to be found at the initial stage of the process, as shown in the example in Figure 16. 


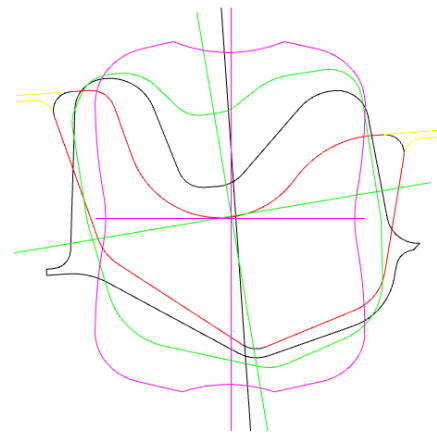

Fig. 16: The three-pass sequence starting with the double symmetric contour to obtain the red-coloured contour identical to the example shown in Fig. 8

The breakdown for each step of the sequence in Fig. 16 is presented in Figures 17 to 20.

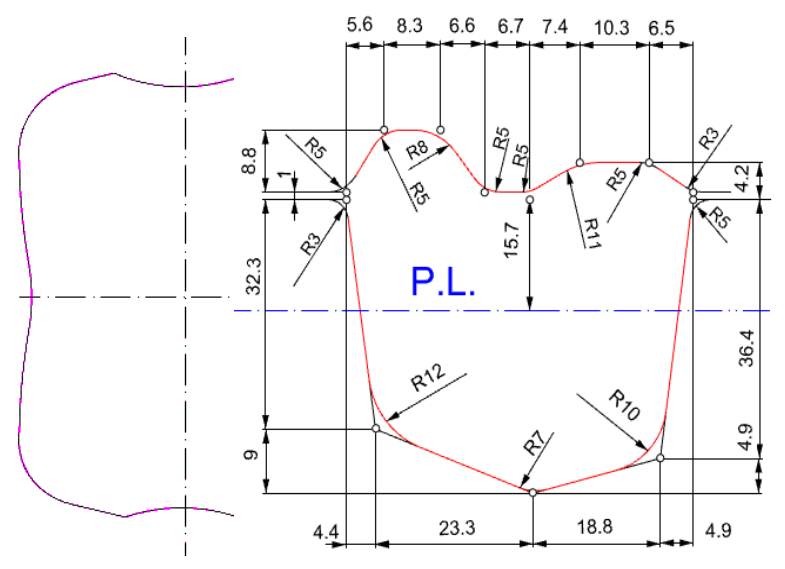

Fig. 17: The double-symmetric entry contour (right) and the first calibre (left)

The centre of gravity of the initial doublesymmetric contour is positioned in the coordinate origin. In the next step, a two-dimensional overlay is developed in which the entering contour is superimposed on the groove contours at the instant of the first contact, Fig. 18.
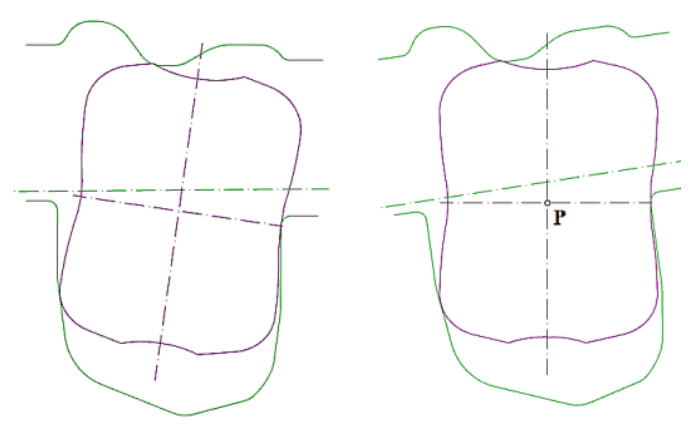

Fig. 18: The instant of the first contact (left), and the position of the $1^{\text {st }}$ calibre after rotating the entry contour about the point $\mathrm{P}$
The locations of the initial contact points are based on mathematical idealisation of gripping the entering contour by two groove contours that are separated by vertical translation from the calibre neutral line. The entering «rigid» cross-section assumes the position that allows for minimising the distance between the grooves under the condition that no part of the involved contours is deformed.

The entering contour is rotated clockwise about the point $\mathrm{P}$, as shown on the left side in Fig 18. It is apparent that this step in positioning the contour is related to the natural tendency of the entering solid to assume the position of the minimum resistance to deformation-i.e., the rolled solid rotates about its longitudinal axis.

On the right side of Fig. 18 the complete overlay is rotated back (counterclockwise). Finally, in Fig. 19 the groove contours are brought back close to each other, still creating the gap at the orifice openings equal to the dimension shown in Fig. 17 (one millimeter in this example).

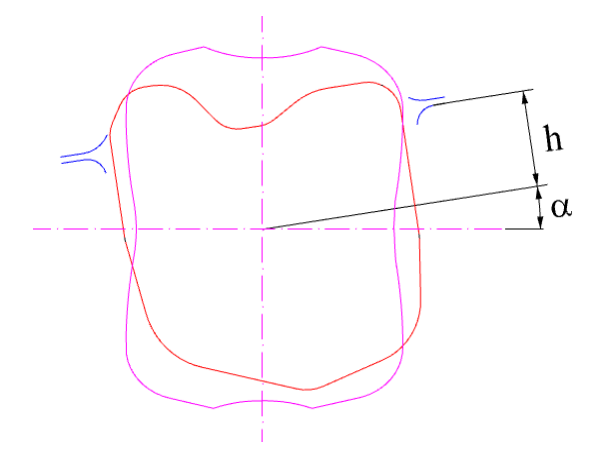

Fig. 19: The resulting position of the orifice (the $1^{\text {st }}$ calibre specified in Fig. 17) in the coordinate system

The coordinate system in which the orifice is positioned is defined relative to the contour of the entering solid. It is apparent in Fig. 19 that the orifice contour is divided by the coordinates into four portions.

The additional translation step, the conversion from the $y-x$ to $x-L$ coordinates, is applied to each of the four portions as discussed in Section 3.3 and demonstrated in Figures 11 and 12.

The digitizing procedure based on Chebyshev polynomials is applied to each of the four portions separately. The resulting vector is composed of the ordered tuple of the Chebyshev coefficients defining all four curves. The Chebyshev transforms are based on deterministic mathematics, i.e. it is only the matter of the finding (by means of increasing) the order $N$ of the Chebyshev polynomials that will satisfy the required precision. The fit at, and only at, the Chebyshev nodes is ideal, and the digital information about the nodes constitutes the vector 
components. Once the group of the orifice geometries of interest is defined, finding the value of $N$ in Eqn. (3) such that all intricacies along the contour are embraced is resolved by increasing the vector dimension.

The orifice design in rolling technology (socalled roll pass design) requires defining the position of the roll gaps as indicated by the bluecoloured details in Fig. 19. These details need to be added to the main contour that was inferred from the analyses of the orifice vectors. However, the related construction technicalities such as the clearance between the rolls and the transition fillet between the roll collar and the groove contour at the roll gap, are matters of well-established roll design routines.

The above procedure is applied to the remaining two passes illustrated in Fig. 16. In this manner, each of the three orifices is positioned in the same coordinate system.

\section{Conclusions}

For most of man-made systems it is impossible to raise the level of sustainability beyond a certain threshold (however unsatisfactory that threshold might be) unless collecting, structuring, digitizing, and analysing the actual observations.

In this manuscript, a new method for digitizing and structuring the morphometric (i.e., pertaining to the geometry and the dimensions) data is presented to address an important aspect of the man-made physical components that comprise orifices enabling various functions of the process. The resulting digitised metrics is better than the existing metrics, since it is better adjusted for analyses and optimisation of the orifice geometry.

The proposed application of Chebyshev polynomials for the preparation the morphometric data has the advantage over the use of one, or a combination of the metaheuristic methods such as Neural Networks, Genetic Algorithms and Fuzzy Logic. This is because the use of the Chebyshev polynomials is based on deterministic formulas and, consequently, leads to the programming and computational simplicity since the digitizing algorithms are based on derivative information. The examples of computational steps are presented more in detail in reference [16]. Moreover, the nowadays availability of computer graphic platforms and big data analytic software allows for combining the data preparation and analytic stages via programming languages such as Python and Java.
Nonetheless, due to the nature of the industrial applications of the orifices, the resulting structured database falls in the category of Big Data because of the quantity of the original unstructured records, and the count of the variables (the database width). Once the geometric data are prepared, the Genetic Algorithms and Fuzzy logic are indispensable in knowledge extraction from the high-velocity Big Data. Moreover, when the patterns, trends and correlations are extracted, each of these methods can be employed for translating the inferences back into the formats of technological documentation such as illustrated in Figures 4, 6, 7, 8, 10, 13 and 17. For example, the inferred correlations are associated with the standard error of estimate, confidence limits etc., and the inferred orifice contour will need to be smoothed or meaningfully repositioned (slightly shifted off the mean position). Here the Machine Learning, where the GA and Fuzzy Logic can be employed is necessary for processing highvelocity Big Data.

The new comprehensive digitized definition of the orifice morphometry allows for unraveling the hidden interrelations and patterns between the orifice entry and exit morphometry along with the interactions and correlations with other factors of the analysed process.

The discussed mathematical definition of the morphometry of critical physical components is an aspect of fundamental importance in the design of Digital Twins. The contemporary concepts of Industry 4.0 and 5.0 have given significant impetus to the development of Digital Twins that will close the loop between the input controls and optimized output by employing artificial intelligence systems. While attracting strong interest from the industry (the market is anticipated to reach over $\$ 15$ billion by 2023) Digital Twin related engineering research is still in its infancy [17]. This provides the additional supporting arguments for the need of the contributions such as presented in this manuscript.

Acknowledgments: This work was supported by the STEM - the University of South Australia https://www.unisa.edu.au/about-unisa/academic-units/stem/ Parts of the presented results are funded by the collaborative research project Transforming Industry Manufacturing Enabler co-financed by the University of South Australia and the Australian Steel Company - Liberty OneSteel. 


\section{References:}

[1] Yan H-S., Information Science: Its Past, Present and Future, Information, Vol.2, No.3, 2013, pp. 510-527.

[2] Gandomi A., Haider M., Beyond the hype: Big data concepts, methods, and analytics, Internationl Journal of Information Managment, Vol.35, No.2, 2015, pp. 137144.

[3] Daru B.H., Park D.S., Primack R.B., Willis C.G., Charles G., Barrington D.S.,. Whitfeld T.J.S, Seidler T.G., Sweeney P.W., Foster D.R, Ellison A.M., Davis C.C., Widespread sampling biases in herbaria revealed from large- scale digitization, The New Phytologist, Vol.217 (2), 2018, pp. 939-955.

[4] Chen, M. et al., Data Mining: An Overview from a Database Perspective, IEEE Transactions on Knowledge and Data Engineering, Vol.8, No.6, 1996, pp. 866883.

[5] Fayyad, U.M., 1996. Data mining and knowledge discovery: making sense out of data, IEEE Expert, Vol.11, No.5, 1996, pp. 20-25.

[6] Teja A.V.R.K, Sri Harsha J., Teja N.S.K., Gangadhar V.S., Sainadh T.H., Static analysis of Orifice Plate for Different Geometries, International Journal Of Engineering And Computer Science, Vol.5, Issue 4, 2016, pp. 16248-16252.

[7] Hanchinal A.M. and Katti V.V., Effect of orifice geometry and orifice to test section spacing on distribution of wall static pressure on a convex surface, Journal of Mechanical Engineering and Sciences, Vol.13, Issue 2, 2019, pp. 4835-4845.

[8] Gaeta R.J., Ahuja K.K., Effect of orifice shape on acoustic impedance. International journal of aeroacoustics, Vol.15 (4-5), 2016, pp.474-495.

[9] Jothi T.J.S., Srinivasan K., Shock structures of underexpanded non-circular slot jets, Sādhanā Vol.44, 25, 2019.

[10] Qamar S.Z., Chekotu J.C., Al-Maharbi M., Alam K.. Shape Complexity in Metal Extrusion: Definitions, Classification, and Applications, Arabian Journal for Science and Engineering, Issue 9, 2019, pp. 1-16.

[11] Kalpakjian S., Schmid S., Manufacturing Engineering and Technology, SI Edition, Pearson Education, 2013.
[12] Mcgannon H.E. (editor), The Making, Shaping and Treating of Steel, United States Steel Corporation, 1971.

[13] Hoonly International Co., (Accessed June $6^{\text {th }}$ 2021) https://haluminium.com/

[14] Roberts W.L., Hot Rolling of Steel (Manufacturing Engineering and Materials), CRC Press, 1983.

[15] Mason J.C., Handscomb D.C., Chebyshev Polynomials, Chapman and Hall/CRC, 2002.

[16] Spuzic S., Translating Morphometric Information About the Rolling Passes Into a Structured Database, Kalibrovochnoe Byuro No.17, 2020, pp. 5-12.

[17] Lu Y., Liu C., I-Kai Wang K., Huang H., $\mathrm{Xu} \quad \mathrm{X}$., Digital Twin-driven smart manufacturing: Connotation, reference model, applications and research issues, Robotics and Computer-Integrated Manufacturing, Vol.61, 2020. 101837.

\section{Creative Commons Attribution License 4.0 (Attribution 4.0 International, CC BY 4.0)}

This article is published under the terms of the Creative Commons Attribution License 4.0 https://creativecommons.org/licenses/by/4.0/deed.en_US 